\title{
Dose Verification System-Radiation Detector for Radiation Therapy
}

\author{
a report by \\ Tina Marie Briere, PhD \\ Department of Radiation Physics, Division of Radiation Oncology, University of Texas MD Anderson Cancer Center
}

How can we be sure that what is planned during the treatment planning phase will be delivered to the patient during the entire course of therapy? Technological gains in radiation therapy, including intensity-modulated radiation therapy (IMRT) and image-guided radiation therapy (IGRT), can be used to create more complex and conformal treatment plans, may deliver higher target doses, and use tighter margins to irradiate smaller treatment volumes and reduce toxicities to normal tissues. While the potential benefits of a correctly delivered plan include better tumor control and lower toxicities to normal tissues, a poorly delivered plan may achieve the opposite outcome. In vivo dosimetry performed within the target volume or close to critical structures can answer the simple but important question: have we delivered the treatment as planned? With the Dose Verification System (DVS), Sicel Technologies (Morrisville, NC, US) has developed the first commercially available permanently implantable metal-oxide-semiconductor field effect transistor (MOSFET) detectors. ${ }^{1-5}$ The detector is designed for daily in vivo dosimetry for megavoltage external beam radiation therapy. While other MOSFET detectors have been used clinically for surface measurements ${ }^{6-9}$ or temporarily placed into a body cavity during treatment, ${ }^{10-12}$ only the DVS detector is designed to be permanently implanted to measure dose over the entire course of treatment. The original prototype DVS detector (see Figure 1a) comprises a single MOSFET within the onboard circuit and an antenna for radiofrequency (RF) communication with an external hand-held reader. The MOSFET is the radio-sensitive element within the detector. Radiation damage increases the gate bias required to allow current to flow, and this increase is proportional to the dose reading. Thus, readings acquired just prior to and immediately following radiation are necessary to compute the measured dose. The detector is fully encapsulated in a biocompatible glass cylinder with a diameter of $3.25 \mathrm{~mm}$ and a length of $25 \mathrm{~mm}$ and is completely wireless. It can be inserted either peri-operatively or percutaneously with a cannula provided by the manufacturer. Because RF telemetry is used to detect voltage changes due to irradiation, these detectors do not require an internal power source. They are unbiased during irradiation, making these passive devices ideal for in vivo use. Unlike other detectors in common use, such as thermoluminescent detectors, photodiodes, and most other MOSFET detectors, the implantable DVS detector is factory-calibrated. (Another type of MOSFET detector, the OneDose, ${ }^{13}$ which is a single-use detector

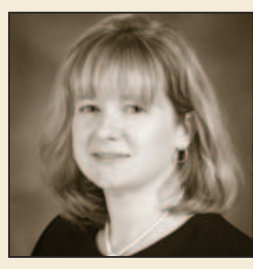

Tina Marie Briere, PhD, is an Instructor in the Department of Radiation Physics at MD Anderson Cancer Center. Prior to her career in medical physics, she was a researcher at the Institute for Materials Research, Tohoku University in Sendai, Japan, performing computations on the electronic and magnetic properties of surfaces and nanomaterials. Dr Briere's current interests include MOSFET detectors, in vivo dosimetry, and respiratory-gated radiation therapy. produced by Sicel Technologies and designed to measure skin dose, is also factory-calibrated.) Dose measurement performed with the RF reader can be completed immediately following treatment, and takes just seconds per detector. These characteristics greatly add to the utility and efficiency of the DVS.

\section{In Vitro Studies-Prototype Detector}

We have performed independent studies to evaluate and assess the performance of the MOSFET itself ${ }^{2}$ and the manufacturer's calibration. ${ }^{5}$ Using an acrylic phantom (Quasar body phantom, Modus Medical Devices, London, Ontario, Canada) under ${ }^{60} \mathrm{Co}$ irradiation, we found the MOSFETs showed a reproducibility to within $\pm 2 \%$ in a fixed geometry and temperature-controlled environment. ${ }^{2}$ Using the factory calibration, we found the dose reproducibility to be within $0.4 \pm 2.1 \%(1 \sigma)$ for the calibration dose of $2 \mathrm{~Gy}$ delivered over 20 fractions. For a larger dose of $4 \mathrm{~Gy}$ per fraction, the dose response was slightly overestimated $(0.9 \pm 2.3 \%(1 \sigma))$, while for a smaller dose of $1 \mathrm{~Gy}$ per fraction, the dose response was slightly underestimated $(-3.3 \pm 3.6 \%(1 \sigma)) .{ }^{5}$ We confirmed the detector's visibility on CT images as well as its relative invisibility on megavoltage portal images. Due to its metal antenna, we expect it to flare on magnetic resonance images. We also suggested that sandwiching two MOSFETs in the circuit would improve the variation in measured dose response throughout the course of treatment. ${ }^{2}$ The manufacturer also performed studies of the detector's performance. ${ }^{1,4}$ When sources of error including set-up, treatment planning, and machine output were considered, the expected performance of the detector was reported to be within $\pm 5 \%{ }^{4}$ Angular variation was found to be within $\pm 1.3 \%$ with respect to rotation about the detector's long axis.' Fading of the voltage reading due to electron-hole recombination ${ }^{14}$ was found to be less than $2 \%$ within 20 minutes.' Temperature variations occurring between the pre- and post-irradiation voltage readings can have a significant effect on the performance of a MOSFET detector. ${ }^{15}$ We found the reproducibility of the detectors to be improved by about $3 \%$ when the temperature was well controlled. ${ }^{2}$ The manufacturer also noted the effect of temperature during in vitro measurements, ${ }^{1,4}$ and suggested this would not be an important issue for in vivo patient use, ${ }^{4}$ as the patient's temperature should not change significantly during a single treatment fraction.

\section{Clinical Studies-Prototype Detector}

The first clinical studies were performed on 10 canine companion animals to monitor adverse events due to detector placement as well as detector migration following implantation. ${ }^{1}$ No adverse events due to implantation were reported. However, repeat CT scans showed migration of about $4 \mathrm{~mm}$ in three of the dogs, and larger migrations of $35 \mathrm{~mm}$ and $70 \mathrm{~mm}$ in two others. This led to the development of a vicryl sock for surgical implantation and a vicryl plug for percutaneous implantation, both of which reduce the chance of migration during the healing process. Clinical studies in humans have also 
We invite you to visit our website, www.mascc.org, to find out about membership, learn about the organization, visit the Education and Research Centers, and to register for our annual international meeting in Houston, Texas, June 26-28, 2008.
The Multinational Association of Supportive Care in Cancer (MASCC), a multinational, multidisciplinary organization, is dedicated to research and education in all aspects of supportive care for people with cancer worldwide regardless of the stage of their disease.
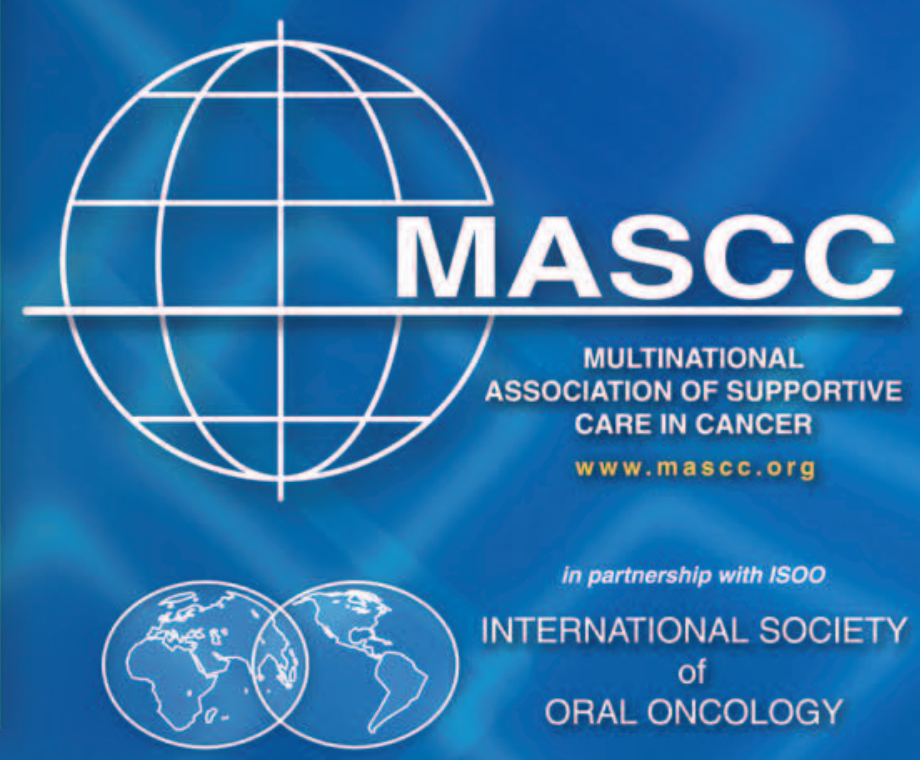

To find out more about MASCC and the Journal of Supportive Care in Cancer please contact Cindy Rittenberg via e-mail at cindyrit@bellsouth.net or visit the Web site at www.mascc.org 
Figure 1: Prototype Dose Verification System Device
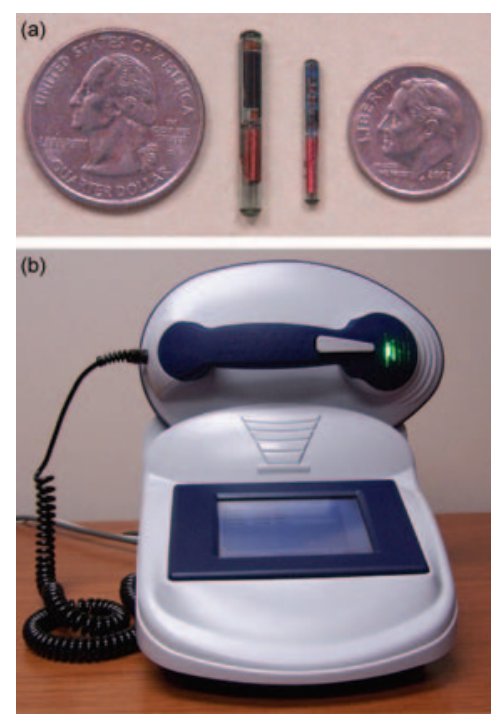

Figure 2: Dose-response Data

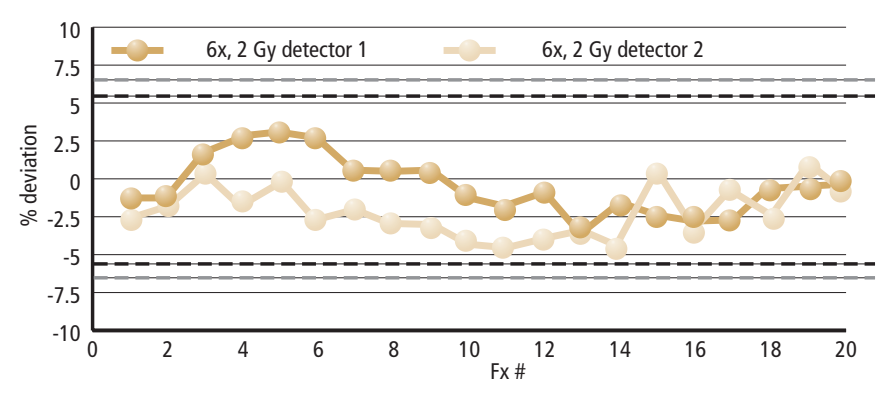

been reported by the manufacturer. ${ }^{3,4,16}$ To date, the original prototype has been successfully implanted into the breast, prostate, rectum, lung, and thigh for in vivo dosimetry during external beam radiation therapy. Of the first 18 patients, ${ }^{4}$ no unanticipated adverse events were reported due to implantation. All detectors performed successfully and only one detector migrated, which was placed in unconsolidated tissue in the lung. The authors reported that 12 of the 18 patients had dose deviations $5 \%$ or larger in at least $40 \%$ of all treatment sessions. ${ }^{4}$ Comparing these results with in vitro phantom measurements with an expected accuracy of $\pm 5 \%$, the authors suggested such deviations could have occurred due to discrepancies with treatment planning (e.g. not using heterogeneity corrections), errors in patient set-up, and patient motion. These results demonstrate the utility of true in vivo measurements with such detectors. The manufacturer has also briefly reported on a larger study involving 60 breast and prostate patients. ${ }^{16}$ Three cases of detector migration $>5 \mathrm{~mm}$ were reported, and again there were no significant adverse events due to implantation. The authors observed significant variation between the predicted and measured dose in at least $33 \%$ of the patients, supporting the results of the initial study.

\section{New ASIC Detector}

The prototype DVS device has recently evolved to a new design (see Figure $1 \mathrm{a})$, the ASIC, which includes a smaller glass capsule $(2.1 \mathrm{~mm}$ width, $20 \mathrm{~mm}$ length) and a double-MOSFET circuit less sensitive to temperature fluctuations. Figure $1 b$ shows the new computer-controlled RF reader. The detectors are factory-calibrated in commercial-sized lots and are intended for daily in vivo dosimetry at body temperature. The manufacturer has specified the dose response to be within 5.5\% (2o) for doses up to 20Gy and 6.5\% (2s) for doses up to 74Gy. ${ }^{17}$ We have performed in vitro measurements to investigate the new detector's performance under 6-MV photon irradiation. Detectors were placed in a water tank at body temperature, and were irradiated to the calibration dose of $2 \mathrm{~Gy}$ per fraction for 20 fractions. As shown in Figure 2, we have found the dose response to be well within the manufacturer's specifications. Improvements to the RF reader design have made the system easier to use within the treatment room environment.

\section{Conclusions}

There is little doubt that the increasing complexity of radiation therapy also increases the cost of error. The development of a true implantable detector is a breakthrough in the field of in vivo dosimetry for radiation therapy, and the DVS manufactured by Sicel Technologies promises to measure dose within the treatment field or in the vicinity of normal tissues with a reasonable accuracy. In practice, we have found the system to be easy to use, permitting multiple detector readings within a matter of seconds. Due to fading of the voltage reading with time, measurements should be taken within 10 minutes of the completion of treatment, which should not be burdensome in a normal clinical environment. True in vivo dosimetry will allow confirmation of treatment delivery and better assessment of normal tissue toxicities. In vivo dosimetry can be used with kilovoltage imaging to provide complementary information, which may allow more accurate assessment for the cause of deviation from the treatment plan, including set-up error and patient motion.

\section{Acknowledgements}

The author has a sponsored research agreement with Sicel Technologies. TMB is grateful to Dr Sam Beddar for his collaboration and mentorship. The author also thanks Michael Riddle and Dr Charles Scarantino.
1. Scarantino $\mathrm{CW}$, et al., An implantable radiation dosimeter for use in external beam radiation therapy, Med Phys, 2004;31:2658-71.

2. Beddar AS, et al., Preliminary evaluation of implantable MOSFET radiation dosimeters, Phys Med Biol, 2005;50(1):141-9.

3. Scarantino $\mathrm{CW}$, et al., Initial clinical results of an in vivo dosimeter during external beam radiation therapy, Int J Radiat Oncol Biol Phys, 2005;62(2):606-13.

4. Black RD, et al., An analysis of an implantable dosimeter system for external beam therapy, Int J Radiat Oncol Biol Phys, 2005;63(1):290-300.

5. Briere TM, Beddar AS, Gillin MT, Evaluation of pre-calibrated implantable MOSFET radiation dosimeters for megavoltage photon beams, Med Phys, 2005;32(11):3346-9.

6. Varadhan $\mathrm{R}$, et al., In vivo prostate IMRT dosimetry with MOSFET detectors using brass buildup caps, J Appl Clin Med Phys, 2006; 7(4):22-32.
7. Dong SL, et al., Estimation of mean-glandular dose from monitoring breast entrance skin air kerma using a high sensitivity metal oxide semiconductor field effect transistor (MOSFET) dosimeter system in mammography, Appl Radiat and Isotopes, 2002;57:791-9.

8. Briere TM, et al., In vivo dosimetry using disposable MOSFET dosimeters for total body irradiation, Med Phys, 2005;32:1996.

9. Scalchi P, Francescon P, Rajaguru P, Characterization of a new MOSFET detector configuration for in vivo skin dosimetry, Med Phys, 2005;32(6):1571-8.

10. Marcié $S$, et al., In vivo measurements with MOSFET detectors in oropharynx and nasopharynx intensity-modulated radiation therapy. Int J Radiat Oncol Biol Phys, 2005;61(5):1603-6.

11. Ciocca M, et al., Real-time in vivo dosimetry using micro-MOSFET detectors during intraoperative electron beam radiation therapy in early-stage breast cancer, Radiother Oncol, 2006;78:213-16.
12. Consorti R, et al., In vivo dosimetry with MOSFETs: Dosimetric characterization and first clinical results in intraoperative radiotherapy, Int J Radiat Oncol Biol Phys, 2005;63(3):952-60.

13. Halvorsen PH, Dosimetric evaluation of a new design MOSFET in vivo dosimeter, Med Phys, 2005;32(1):110-17.

14. McLean FB, Boesch HE, Oldham TR, Electron-hole generation, transport, and trapping in $\mathrm{SiO2}$, in lonizing radiation effects in MOS devices and circuits, TP Ma and PV Dressendorfer, 1989;87-188.

15. Haran A, et al., Temperature effects and long term fading of implanted and unimplanted gate oxide RADFETs, IEEE Trans Nucl Sci, 2004;51(5):2917-21.

16. Scarantino $\mathrm{CW}$, et al., True in vivo dosimetry in breast and prostate cancer patients: Variance between predicted and measured dose, Int J Radiat Oncol Biol Phys, 2006;66(3):S104-5.

17. Sicel Technologies Inc., Private communication, 2007. 